\title{
Development of an Awaking Behavior Detection System with Kinect
}

\author{
Hironobu Satoh ${ }^{1}$ Kyoko Shibata ${ }^{2}$ and Tomohito Masaki \\ ${ }^{1}$ Kochi National College of Technology \\ Monobeotsu 200-1, Nangoku, Kochi, Japan \\ 2 Kochi University of Technology \\ Miyanokuchi 185, Tosayamada, Kami, Kochi, Japan \\ satoh@ee.kochi-ct.ac.jp, shibata.kyoko@kochi-tech.ac.jp \\ http://www.kochi-ct.ac.jp, http://www.kochi-tech.ac.jp
}

\begin{abstract}
The purpose of this study is detecting unsafe behavior of the person lying on the bed and warning caregivers that a person is falling down from the bed.

However, in the dark room at night, the detection ability of previous system is low, because the brightness adjustment processing of a Web camera is not able to adjust brightness of the dark room.

In this paper, we propose a new detection system using Kinect. Kinect has a depth sensor consisted of infrared leaser. And, Kinect is able to measure distance between Kinect and an object in the dark room. Moreover, the behavior of an old person is extracted from measured data by Kinect. By using Kinect, it is considered that the awakening behavior detection system is able to be used in the dark room at night.

In this paper, the awakening detection system using Kinect is shown. And, in experiment, the capability of the proposed system have been verified.

From the result of the experiment, the detection rate of the safe behavior have been $94 \%$. And, the detection rate of the unsafe behavior have been $80 \%$.
\end{abstract}

Keywords: Awaking behavior detection system, Neural network, Kinect.

\section{Introduction}

Old people, which need a nurse, sometime fall down from a bed and broke their thighbone. To avoid this accident, it is necessary to care for old people during a day by caregivers. However, to care during a day is a great burden for caregivers. Then, we have proposed an awakening behavior detection system that watches an old person during a day by artificial intelligence. And the awakening behavior detection system warns caregivers that an old people are falling down from the bed.

Our previous research, in the awaking behavior detection system, a Web camera is used for capturing the behavior of an old people. It is known that unsafe 
behavior is different between individual old people. Therefore, NN (Neural Network) detect unsafe behavior of old people using capturing images 122345 .

However, in the dark room at night, the detection ability of previous system is low, because the brightness adjustment processing of a Web camera is not able to adjust brightness of the dark room. It is a problem that the previous system is not able to use in the dark room at night.

In this paper, we propose a new detection system using Kinect, which is developed by Microsoft for human computer interface. Kinect has a depth sensor consisted of infrared leaser. And, Kinect is able to measure distance between Kinect and an object in the dark room. Moreover, the behavior of an old person is extracted from measured data by Kinect. By using Kinect, it is considered that the awakening behavior detection system is able to be used in the dark room at night.

In this paper, the awakening detection system using Kinect is shown. And, in experiment, the capability of the detecting unsafe behavior of the proposed system is verified.

\section{Problem of the Previous Research}

In the previous research, the detection algorithm is shown as follow. The person lying on the bed is captured by Web camera. Then, the captured image is transformed into the input data to NN as follows. The captured picture is transformed into the gray-scale picture. The picture is divided into $200(20 \times 10)$ blocks. Each block is transformed into a binary value, 0 or 1 , depending whether the arithmetic mean of the values of the block is larger than a threshold or smaller. These binary values are used as the input data to NN 6]. By using NN, the system can learn each person's behavior easily and adapt user's peculiar behaviors.

The capability of the awakening behavior detection system is shown. Therefore, in the dark room, the capability of the proposed system is low. Because, the brightness adjustment included in Web camera is not able to adjust brightness of the dark room. So, the target subject is not able to be captured clearly. Then, the previous system is not able to detect the target behaviors in the dark room at night.

\section{Proposed System}

\subsection{Kinect}

Thereupon, in this paper, we propose new detection system using Kinect which is one of a human computer interface. In the proposed system, Kinect is placed near the ceiling and directly above the bed. Fig[1] shows the installation example of the Kinect. The proposed system using Kinect is able to measure the distance between Kinect and some objects in the dark room. The measured distance is used for determining the figure of the person lying on the bed. 


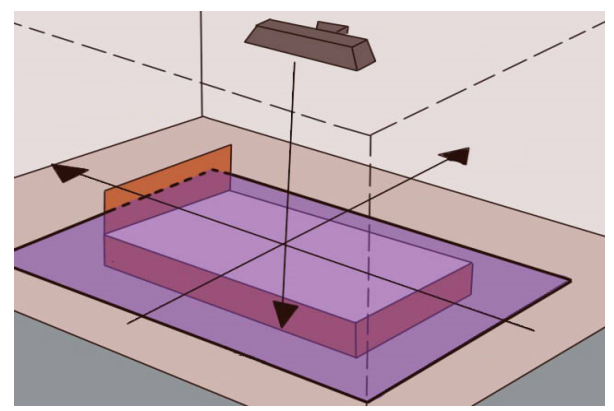

Fig. 1. Installation of Kinect

\subsection{Preprocessing}

The detection algorithm of the proposed system is shown as follow. First, the distance is measured between the bed and Kinect. And the measured distance is defined as threshold for detecting a person lying on the bed. As preprocessing, person's figure is detected using threshold. It is defined as a person's body each pixel of measured data by Kinect that the distance is closer than the threshold. Fig 2 illustrates the image of the person's body detection, when threshold value is changed. Fig 3 shows the detected figure of a person. As the figure shows, person's sharp is detected. By optimizing the threshold value, the shape of a human is able to be detected correctly. Pixels, which are detected as person's body, are converted to values as 0.98 . And, other pixels are converted to values as 0.1 (Fig 3).

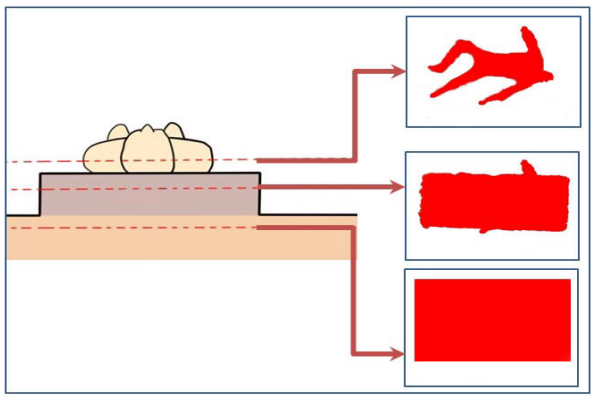

Fig. 2. Image of a human body detection 


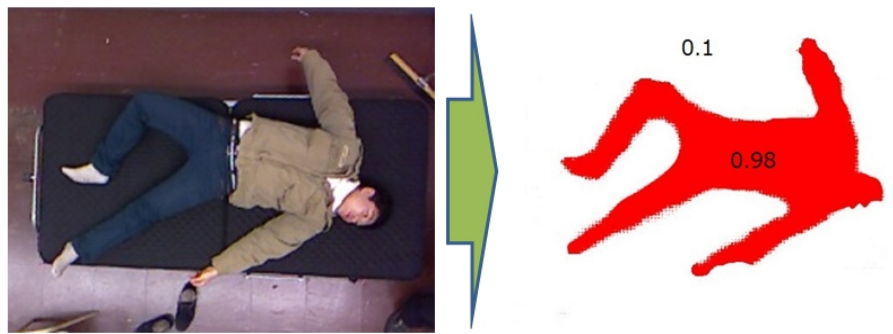

Fig. 3. Figure detection and converted values

\subsection{Detection}

Then, converted values are inputted to DBN (Deep belief networks) 7] beforehand executed learning. DBN is known as a one of the deep learning. In the research field of speech recognition and image recognition, high recognition ability of DBN has been confirmed. DBN learn the safe behavior and unsafe behavior using converted values. DBN recognize the behavior of the person lying on the bed as safe behavior or unsafe behavior.

\section{Experiment}

In this section, the capability of the proposed system using Kinect is verified. The purpose of this experiment is to verify the capability of the detection ability of the proposed system. Thereupon, a coverlet was not putted on the subject. A state of lying on the bed was defined as a safe behavior. Fig2 is an example of safe behavior. A state of falling down was defined as an unsafe behavior. Fig 4 summarizes an example of unsafe behavior. In the experiment, at the first, safe behaviors and unsafe behaviors of the subject ware captured by Kinect and

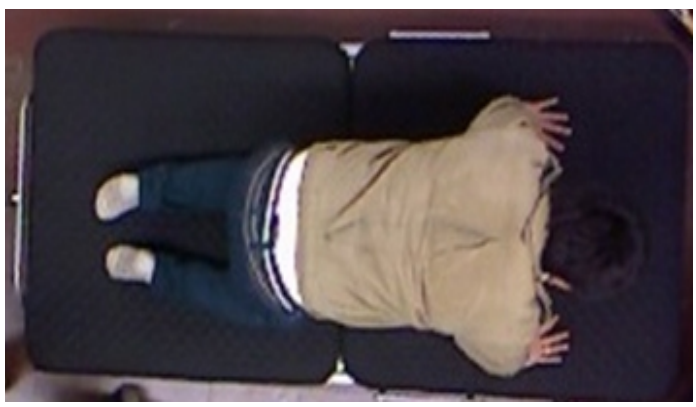

Fig. 4. Example of the unsafe behavior 
stored on the personal computer. From the stored data, 250 data each behavior ware used for learning. And, 100 data each behavior ware used for evaluating as unknown data. Learning have been executed while 7 days on personal computer, which consist of intel xeon $3.0 \mathrm{GHz}$, 4core.

\section{$5 \quad$ Results and Discussions}

The rate of the safe behavior has been $94 \%$. And the rate of the unsafe behavior has been $80 \%$.

From the results, the capability of the proposed awaking detection system is practical. In this experiment, verification of the detection ability has been executed without the coverlet. The coverlet is usually put on the old person. In the feature, it is necessary that the experiment is executed, while the subject are put coverlet.

\section{Conclusion}

The detection ability of the awaking behavior detection system using Web camera is low in the dark room at night. Accordingly, we have proposed a new system using Kinect. In the experiment, it is confirmed that the proposed system is able to recognize the safe behavior and unsafe behavior of the subject lying on the bed.

\section{References}

1. Ikeda, R., Satoh, H., Takeda, F.: Development of Awaking Behavior Detection System Nursing Inside the House. In: International Conference on Intelligent Technology 2006 , pp. 65-70 (2006)

2. Matubara, T., Satoh, H., Takeda, F.: Proposal of an Awaking Detection System Adopting Neural Network in Hospital Use. In: World Automation Congress 2008 (2008)

3. Satoh, H., Takeda, F., Shiraishi, Y., Ikeda, R.: Development of a Awaking Behavior Detection System Using a Neural Network. IEEJ Trans. EIS 128(11), 1649-1656 (2008)

4. Yamanaka, N., Satoh, H., Shiraishi, Y., Matsubara, T., Takeda, F.: Proposal of The Awakening Detection System Using Neural Network and It's Verification. In: The 52nd The Institute of Systems, Control and information Engineers (2008)

5. Satoh, H., Takeda, F.: Verification of the Effectiveness of the Online Tuning System for Unknown Person in the Awaking Behavior Detection System. In: Omatu, S., Rocha, M.P., Bravo, J., Fernández, F., Corchado, E., Bustillo, A., Corchado, J.M. (eds.) IWANN 2009, Part II. LNCS, vol. 5518, pp. 272-279. Springer, Heidelberg (2009)

6. Haykin, S.: Neural Networks a comprehensive foundation, New Jersey, USA, pp. 161-173 (1998)

7. Yoshua, B., Pascal, L., Dan, P., Hugo, L.: Greedy Layer-Wise Training of Deep Networks. In: Advances in Neural Information Processing Systems 19, pp. 153-160 (2006) 